\title{
Developing a systematic approach to the analysis of time-varying main-bearing loads for wind turbines
}

\author{
Edward Hart ${ }^{1 *}$
}

${ }^{1}$ Wind Energy and Control Centre, Electronic and Electrical Engineering, University of Strathclyde, Glasgow, UK

\section{Correspondence}

*Edward Hart, Wind Energy and Control Centre, Electronic and Electrical Engineering, University of Strathclyde, Glasgow, UK

Email: edward.hart@strath.ac.uk

This work was funded by the EPSRC (grant no. EP/R513349/1).

\begin{abstract}
This paper considers the time varying loads experienced by wind turbine main-bearings. Radial load trajectories based on simulated data indicate that 'looped' structures, in the form of repeating changes in radial load magnitudes and directions which form elliptical patterns, are present which have not before been described in the literature. In order to allow these identified structures to be described and studied, an automated method for identification and parameterisation of these loops is presented, along with preliminary results from applications on simulated data. In order to assess the relative importance and potential impacts of these identified structures on bearing rollers, an internal load model for double-row spherical roller bearings is also developed. Results indicate that identified loops in radial main-bearing applied loads lead to significant fluctuations in bearing roller loads, even within normally unloaded regions. These findings motivate further study with respect to the identified load structures and their impacts on wind turbine main-bearing internal loading and failures. The methods developed in this paper provide the basis for a systematic approach and necessary tools for this future work.
\end{abstract}

KEYWORDS:

Wind turbine, main-bearing, loading, Hertzian contact model

\section{1 | INTRODUCTION}

Wind turbine main-bearings have been identified in the literature as a component for which more research focus is needed owing to relatively high reported failure rates ${ }^{1}$. In Hart et. al. $(2020)^{2}$ it is argued that the full chain of loads dynamics, from the wind field and aerodynamic interactions through to individual roller loads and damage, should be considered in order for this component to be properly understood. This way, contributing factors to main-bearing failures from the full envelope of wind turbine design, operational decisions and control can be considered to seek solutions in this whole-system context. Key to the this approach will be a detailed consideration of time-varying loads experienced by wind turbine mainbearings, which forms the central topic of this paper. At this stage, the key factor or factors leading to premature main-bearing failures remain unknown. In addition, the complexity of this particular problem is high since the main-bearing lies at the interface of a number of disciplines due to its location and role in wind turbine systems. Looking ahead, a full resolution of the main-bearing problem will require much detailed modelling for a range of dynamic and possibly damaging effects, comprehensive studies correlating failure data with loading, wind field structure, design, tribological factors (e.g. lubrication) and operational/control effects; as well as measurement campaigns on operational turbines to validate findings and explore additional real world effects. In order to approach this topic systematically, improve our current understanding and help guide research focus in this area, this paper looks to 1) understand the characteristics and structure of wind turbine main-bearing loading 2) develop methods for analysing and parametrically describing observed loading behaviours 3) explore the impacts of observed loading on main-bearing rollers to consider possible damaging effects and demonstrate the importance of identified structures and the utility of the presented methods. 
Section 2 considers time-varying radial loads applied to the main-bearing using simulated turbine data. Additional structure is shown to be present in these loads which takes the form of repeating looping patterns in the radial load vector trajectories. Section 3 develops a method for autoidentification and classification of these loops, along with preliminary results considering prevalence and characteristics of loops across simulated data. In order to assess the relative impacts of identified load structures, while also providing an accessible bearing load model for applications in this field more broadly, Section 4 presents an internal load model for spherical roller bearings which accounts for the presence of both radial and axial (i.e. thrust) loading. Emphasis is placed on simplicity and modularity with respect to this model, allowing assessment of identified load structures without the high complexities inherent in more sophisticated models ${ }^{3456}$, while also ensuring the developed model can be extended in future work to include additional effects as required. In Section 5 this model is applied to consider the relationship between identified load loops (and loading more generally, including thrust), fluctuations in main-bearing roller loads and the possible impacts of such effects. It is demonstrated that radial loading is the main driver for main-bearing roller load fluctuations.

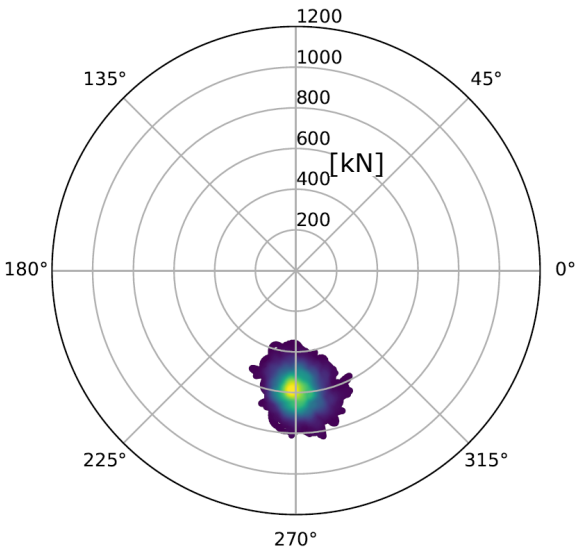

(a) Mean wind speed $=6 \mathrm{~m} / \mathrm{s}$

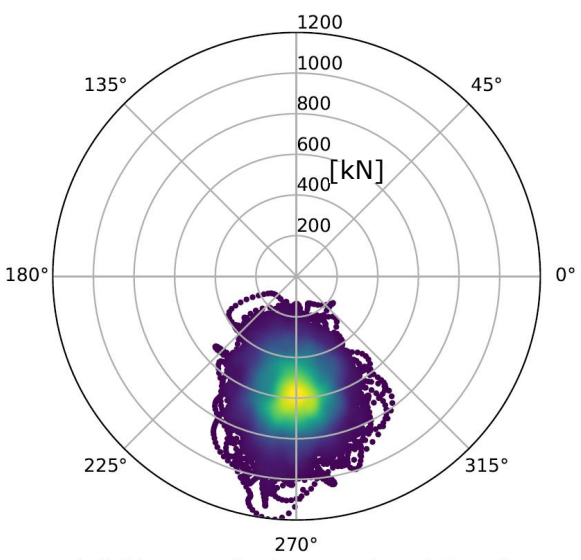

(c) Mean wind speed $=18 \mathrm{~m} / \mathrm{s}$

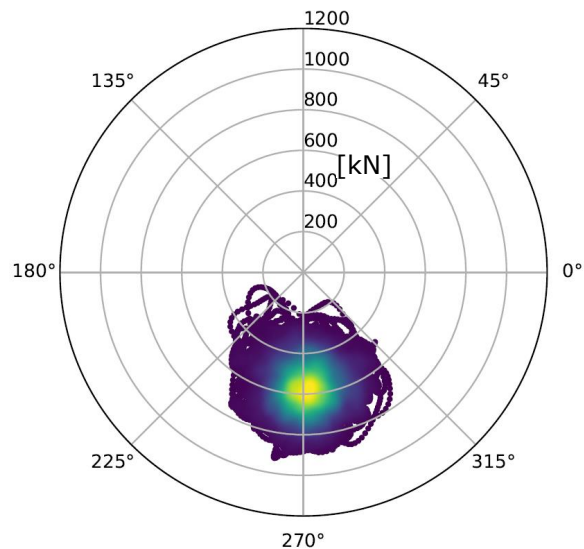

(b) Mean wind speed $=12 \mathrm{~m} / \mathrm{s}$

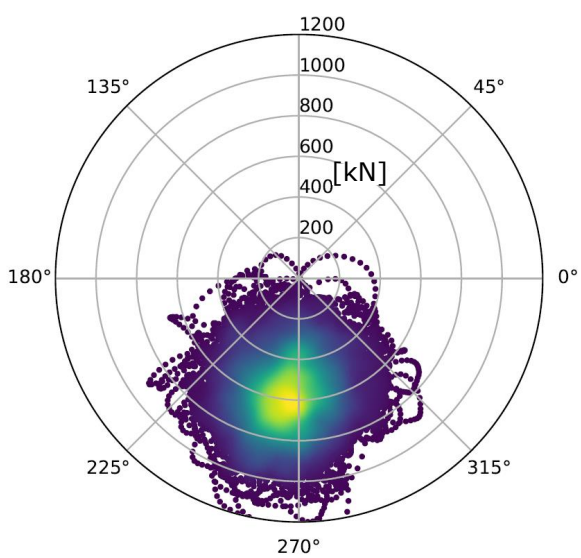

(d) Mean wind speed $=24 \mathrm{~m} / \mathrm{s}$

FIGURE 1 Main-bearing radial applied load density plots from 10 minute aeroelastic simulations in turbulent wind fields with mean wind speeds ranging from 6 to $24 \mathrm{~m} / \mathrm{s}$.

\section{2 | TIME-VARYING MAIN-BEARING LOADS}

To illustrate findings and develop the methods in the current paper, hub loads were generated from simulations in turbulent wind for a $1.5 \mathrm{MW}$ variable-speed, variable-pitch wind turbine using DNV-GL bladed software and inputted into the single main-bearing model of Hart et. al. (2019) 1 
in order to generate a collection of 10min main-bearing applied load time-histories Using this data, main-bearing loads were studied visually as an initial step to moving beyond the summary values explored in previous work ${ }^{1}$. Figure 1 shows density plots for the radial loads applied to the main-bearing by the low speed shaft throughout $10 \mathrm{~min}$ simulations in turbulent wind fields with given mean wind speeds and medium turbulence levels, as defined by the IEC standards. Note, these plots are showing the applied load when looking towards the turbine from the upwind side and show both direction and magnitude of the radial force. As would be expected, these load distributions are each centered about the rotor weight in the vertical direction. It is clear that variations in radial loads increase dramatically with wind speed. Furthermore, the visible fluctuations about the edges of these distributions indicate that additional dynamic structure may be present. Moving from distributions to trajectories in time, large repeating looped patterns become apparent throughout much of the load time-history. Examples of these are shown in Figure 2 taken from 18 and $24 \mathrm{~m} / \mathrm{s}$ mean wind speed cases. Given that these load trajectories are only $2.4 \mathrm{~s}$ in length, the main-bearing is experiencing highly fluctuating loads in both magnitude and direction. Load values can be seen to half and then return to their original value in a matter of seconds and a load reversal event, where gravitational forces are overcome and the rotor is being 'lifted', is also present. Figure 2 (c) demonstrates the presence of repeating patterns of similarly sized and shaped loops which have been observed throughout these load time histories. Assuming such loops are indeed present throughout much of the time a wind turbine is operational, they would seem to warrant proper attention in terms of their underlying causes, characteristics and effects on bearing internal loads. Fluctuations in loading of this sort could be a cause of surface fatigue, however, such rapid changes in load magnitudes and directions might also be leading to other forms of damage. In the main-bearing context it has previously been suggested that reported rates of failure could be indicative of non-fatigue damage modes, such as abrasion and wear, playing a role 1 . The presence of these load structures could therefore be important in further exploring those possibilities. It is therefore proposed that a methodology is required which allows for individual load loops to be identified and characterised such that their prevalence, size and associated attributes can be analysed across datasets, ideally using parametrised quantities for easy analysis. A roller load analysis using a wind turbine main-bearing model should then be performed in order to assess the effects of loops identified with the developed method on roller loading; in particular, with a view to understanding possible impacts (both fatigue and non-fatigue related) to main-bearing health, considering whether these load structures are still important in the presence of thrust loading, and identifying required future work as indicated by findings. These listed aims form the scope of the current paper.

\section{3 | AUTOMATED IDENTIFICATION OF LOAD STRUCTURES}

This section presents a method by which potential loops in main-bearing loads can be identified. Let $\mathbf{G}_{r}(t)$ be the radial load vector applied to the main-bearing by the low speed shaft at time $t$. Tangent lines to the curve formed by $\mathbf{G}_{r}(t)$ lie at some angle, $\theta$, to the horizontal at each time-step. A new function $\theta(t)$ is therefore defined which equals this tangent line angle for each value of $t$. Considering two consecutive times, $t_{1}$ and $t_{2}$, and the force trajectory on this portion of the curve, one can interpret the angle moved through by $\mathbf{G}_{r}$ from $t_{1}$ to $t_{2}$ as being,

$$
\Delta \theta_{t_{1}, t_{2}}=\theta\left(t_{2}\right)-\theta\left(t_{1}\right)
$$

An example of this is shown in Figure 3 The cumulative angle moved through by the load trajectory, denoted here by $\Theta$, can then be tracked using a summation of these terms. Letting $\tau$ denote time-step, the cumulative angle from $t_{0}$ to $t_{*}$ is defined as,

$$
\Theta_{t_{0}, t_{*}}=\sum_{t=t_{0}}^{t_{*}-\tau} \Delta \theta_{t+\tau, t} .
$$

Given a closed loop, it should be clear that the cumulative angle around that loop will be equal to $\pm 2 \pi$, with the sign accounting for clockwise or anti-clockwise travel. Analogously, a potential loop starting from time $t_{0}$ is defined as being the portion of the curve $\mathbf{G}_{r}(t)$ lying between $t_{0}$ and $t_{*}$, where this latter time is the first after $t_{0}$ such that both of the following conditions hold:

$$
\begin{gathered}
\left|\Theta_{t_{0}, t_{*}}\right| \leq 2 \pi \\
\left|\Theta_{t_{0}, t_{*}+\tau}\right|>2 \pi .
\end{gathered}
$$

This allows for a time-series of loading to be broken up into constituent potential loops by applying the above formulation at the beginning of the load time-history, identifying the end time, $t_{*}$, of the current potential loop and then repeating the process starting from the next time-step.

Figure 4 shows examples of identified potential loops using this method. It has been found that there are three distinct types of potential loop: full loops - what one would intuitively identify as loops and with end points overlapping or lying close together, partial loops - similar to full loops but ending prematurely with a sizeable gap between endpoints, non loops - more akin to random fluctuations. In addition to parametrically describing

\footnotetext{
${ }^{\dagger}$ In the previous paper it is main-bearing reaction loads that are extracted, and so the applied loads used here are the negative of those in this previous work (since they act in opposite directions).
} 


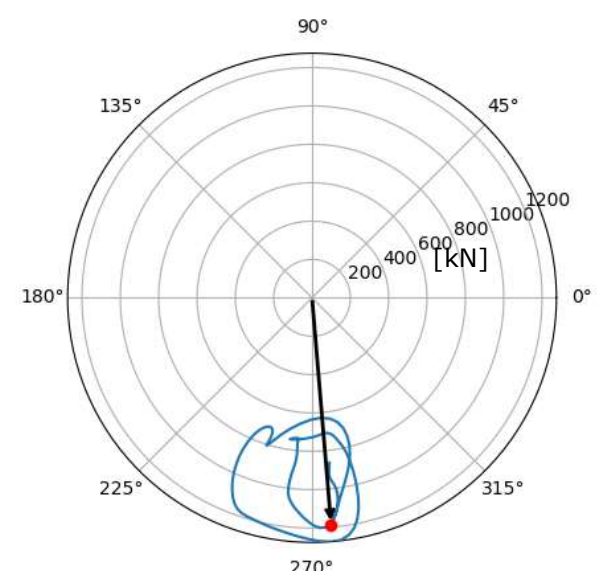

(a)

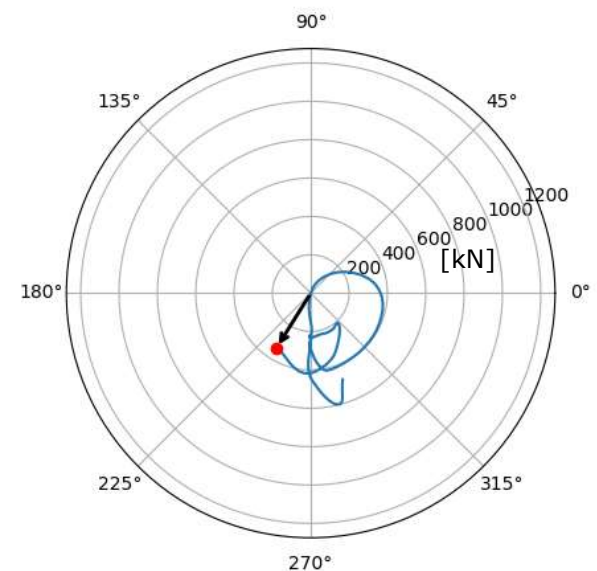

(b)

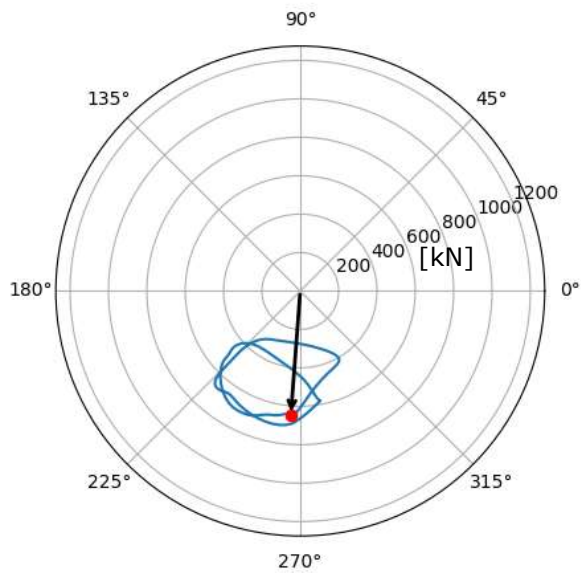

(c)

FIGURE 2 Load loop examples from simulated data for a 1.5MW wind turbine. The arrows give instantaneous load and the blue curves trace their trajectories over the previous $2.4 \mathrm{~s}$.

loops, it is therefore also necessary to be able to classify potential loops into the categories listed here such that each can be identified. While non loops will certainly play a role in main-bearing health, this work will focus more on the highly structured full and partial loop cases. It transpires that parameterisation and classification of loop cases can be achieved in parallel.

\section{1 | Ellipse fitting for loop parameterisation}

The example full and partial loops in Figure 4 appear roughly elliptical in shape and the same has been found across identified loops in general. Ellipse fitting is therefore considered as a way of providing a parametric description of these load structures. This is an attractive option since an ellipse has associated with it measures of size (area) and shape (eccentricity) while elliptical fits result in all loops being described using a common set of parameters. The technique used in the current work is presented in Fitzgibbon et. al. (1996)7. A brief overview of this method is given in Appendix A Examples of ellipses fitted to full loop cases using this method are shown in Figure 5

\subsection{Classifying full, partial and non loop cases}

While the proposed approach of using elliptical fits to parameterise load structures is attractive, in order to be useful it will also be necessary for the various loop cases (full, partial, non) to be distinguished. While a range of approaches could be taken to this classification task, the method developed here was borne out of the following intuitive observations which become evident after elliptical fits are applied to various examples:

1. Full loops conform well to the elliptical fit around the full circumference 


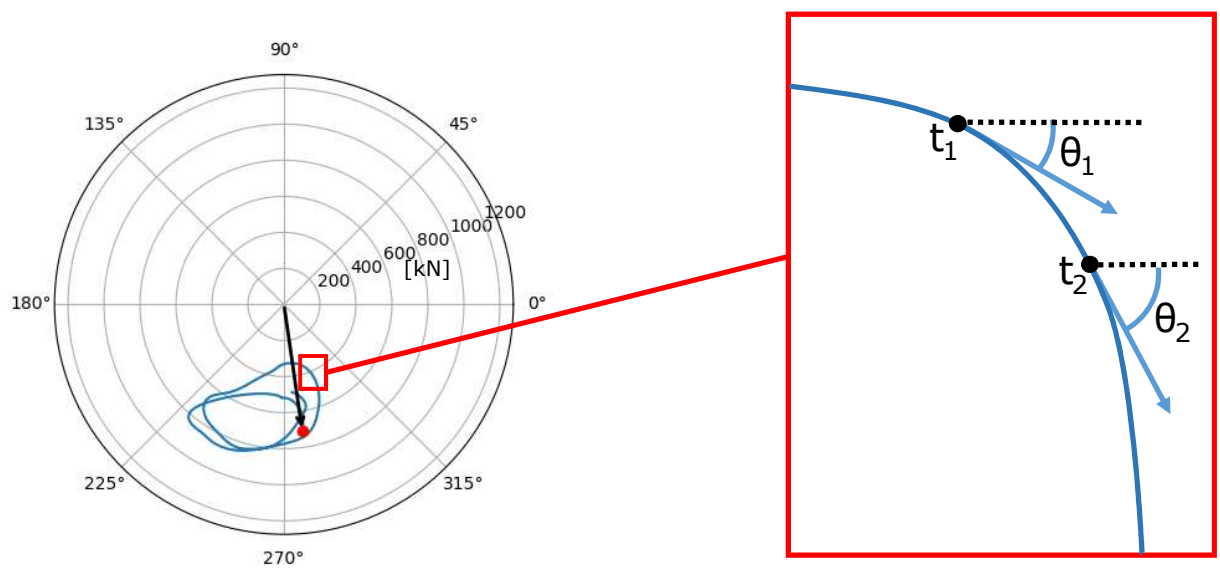

FIGURE 3 Load trajectory tangent lines and angles used to compute the cumulative angle with which potential load loops are identified.

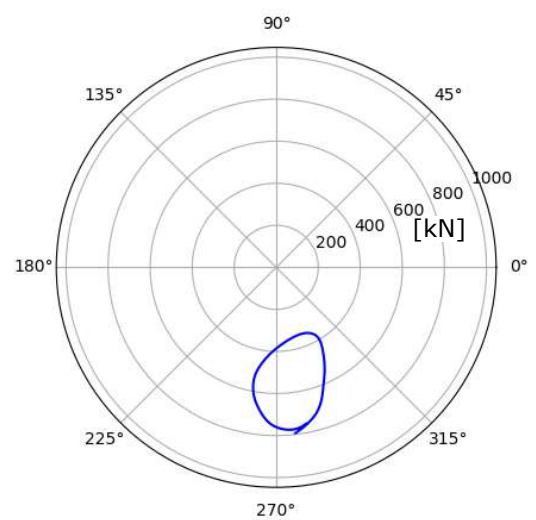

(a)

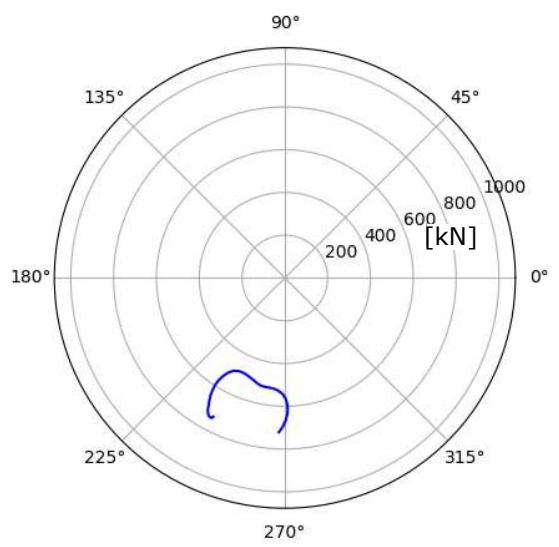

(b)

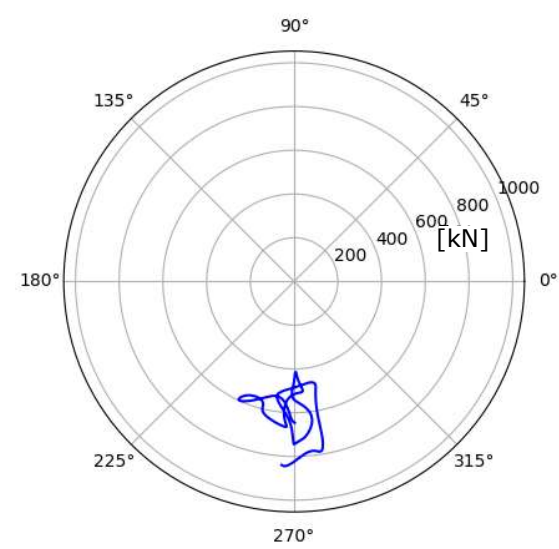

(c)

FIGURE 4 Examples of identified potential loops. Note that these fall into three obvious categories of full loops (a), partial loops (b) and non loops (c).

2. Partial loops conform well to the elliptical fit where data is present, but, a gap is present a portion of the circumference

3. Non loops conform badly to the elliptical fit around the full circumference with data often also in the ellipse interior.

These observations indicate that a classification based on goodness-of-fit measures may be viable, and a two-stage goodness of fit classification has been developed for this purpose. 


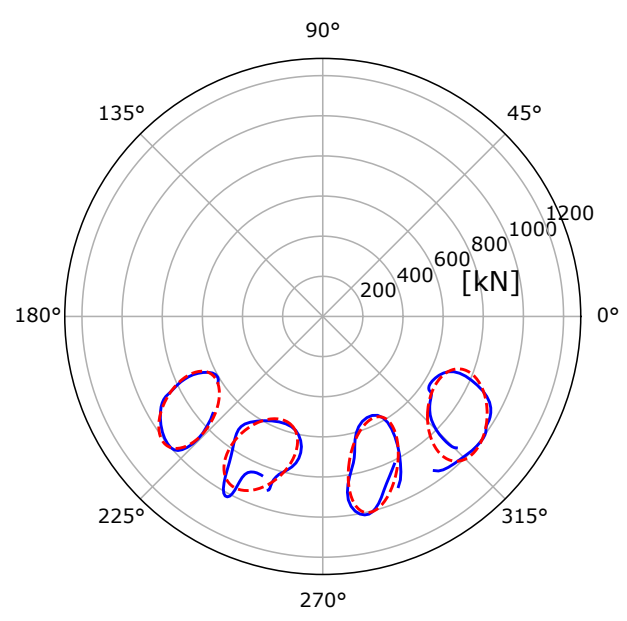

FIGURE 5 Examples of identified full loops and their fitted ellipses.

The coefficient of determination, commonly denoted $R^{2}$, describes the proportion of variance present in a set of measured values which is captured by a model fitted to the data. The $R^{2}$ value for a model $\mathbf{Q}$ as a fit to data $\mathbf{P}$ is given by,

$$
R^{2}(\mathbf{Q}, \mathbf{P})=1-\frac{S_{\text {resid }}(\mathbf{Q}, \mathbf{P})}{S_{\text {total }}(\mathbf{P})}
$$

where $S_{\text {resid }}$ and $S_{\text {total }}$ are the residual and total sum-of-squares values respectively, defined explicitly in Appendix $B$ Let $\mathbf{L}$ be the set of points in a given potential loop we wish to classify. If $\mathbf{E}$ is then the elliptical model for $\mathbf{L}$, defined such that for each point in $\mathbf{L}$ the corresponding entry in $\mathbf{E}$ is the closest point on the fitted ellipse to the given data point, it follows that the coefficient of determination for $\mathbf{E}$ as a fit to $\mathbf{L}$, denoted here by $R_{A}^{2}$, is,

$$
R_{A}^{2}=R^{2}(\mathbf{E}, \mathbf{L}) \text {. }
$$

This value, which has a maximum of 1 , can be interpreted as a goodness-of-fit score for how well the data is described by the ellipse. Notice that when calculating $R_{A}^{2}$, it is the locations of datapoints in $\mathbf{L}$ which drive where goodness-of-fit is scrutinised. It follows that this score cannot, in isolation, be used to differentiate between full and partial loop cases since, in the latter, the 'gap' in measured data with respect to the ellipse is not taken into account. This issue has been solved by defining a second goodness-of-fit score, denoted $R_{B}^{2}$, which can be used in conjunction with $R_{A}^{2}$ to delineate between loop cases. Conceptually, where $R_{A}^{2}$ was constructed by taking each measured data point in turn and asking "how close is the nearest point on the ellipse?", $R_{B}^{2}$ will instead be defined such that it is taking each point around the ellipse in turn and asking "how close is the nearest point in the measured data?".

Assume that a potential loop $\mathbf{L}$ has had an ellipse fitted to it, resulting in a set of elliptical parameters, $\sigma$, with which the values on the ellipse can be calculated at any angle around the circumference. $\widetilde{\mathbf{E}}$ is then defined to be the set of points on the ellipse taken around the full circumference at a number, $m$, of equally spaced angles between 0 and $2 \pi$. Throughout this work a value of $m$ is used which is equivalent to $1^{\circ}$, so $m=\pi / 180$ rad. A new set, $\widetilde{\mathbf{L}}$, is also defined such that: for each point in $\widetilde{\mathbf{E}}$, the corresponding point in $\widetilde{\mathbf{L}}$ is the measured data point (from $\mathbf{L}$ ) which lies closest to the given point on the ellipse. With the above definitions in place, $R_{B}^{2}$ can be formally defined as,

$$
R_{B}^{2}=R^{2}(\widetilde{\mathbf{L}}, \widetilde{\mathbf{E}}) \text {. }
$$

Along with the newly defined sets being used in this second goodness-of-fit equation, it should be observed that the order in which they are entered into the $R^{2}$ equation has also changed. This is because, in order to have a measure which can help account for possible gaps in loop data, this latter measure is treating the data as a fit to the ellipse and, in doing so, uses the full circumference of values around the ellipse when scrutinising goodness-of-fit. This formal definition can be seen to conform to the conceptual description of $R_{B}^{2}$ given above. Figure 6 presents a graphical depiction of the two $R^{2}$ measures defined here. The full, partial and non loop cases are now considered with respect to the defined goodness-of-fit measures. Along the same lines as observations made previously:

1. For a full loop which is fitted well by an elliptical model high values would be expected for both $R_{A}^{2}$ and $R_{B}^{2}$

2. For a partial loop which is fitted well by an elliptical model where data is present a high value would be expected for $R_{A}^{2}$, while a low value would be expected for $R_{B}^{2}$

3. A non loop would be expected when neither of the above cases hold. 


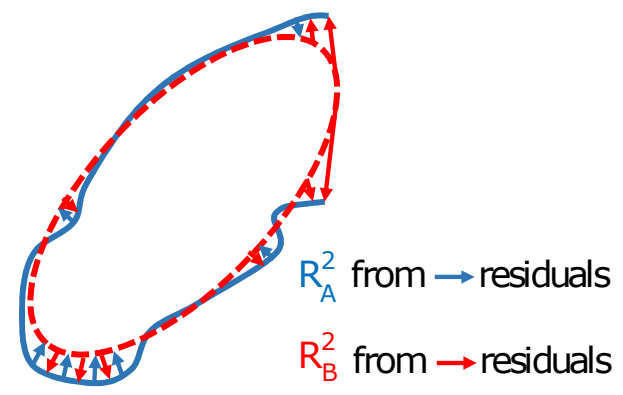

FIGURE 6 Graphical depiction of residuals seen by the two applied goodness-of-fit measures between measured data (solid blue curve) and a fitted ellipse (dashed red).

The above is implemented in practise by selecting an $R^{2}$ threshold value, $\eta$, and applying Algorithm 1 A value for $\eta$ must be selected to apply this

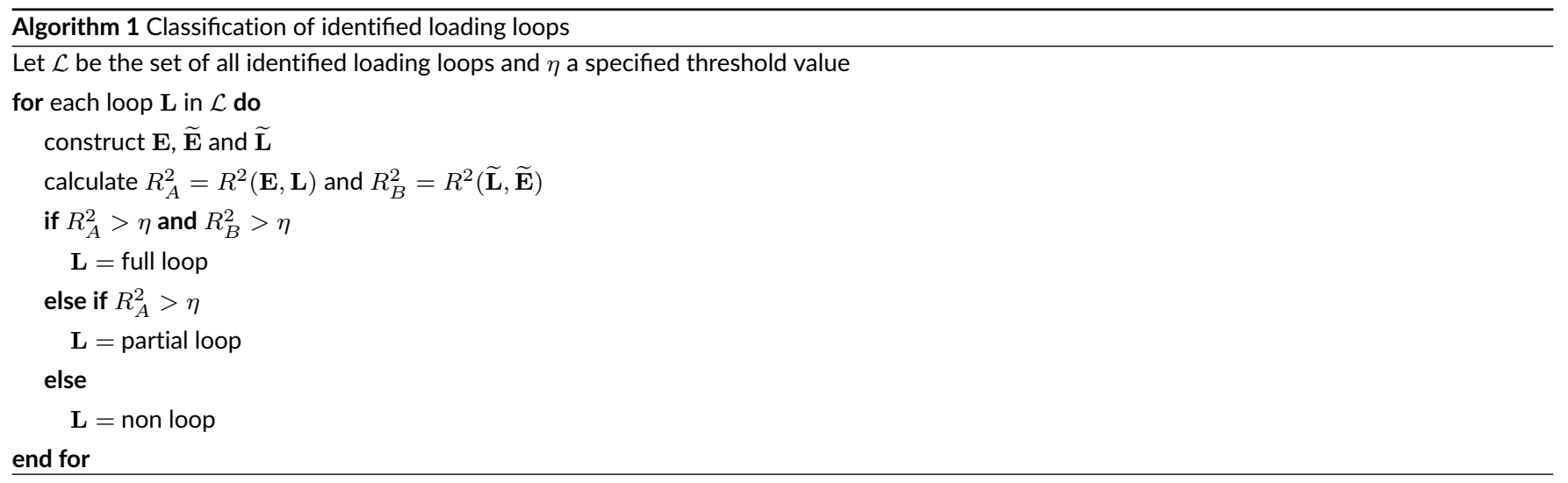

approach. In practise, $\eta$ must be set through a process of observing large numbers of fits and their goodness-of-fit measures. The reason for this is, if one orders all loops in terms of ascending goodness-of-fit, the $\eta$ value effectively decides the cutoff between good and bad on this scale. If it transpired there was a gap in fit quality values there might be a natural point at which to set $\eta$, but, in reality there is very much a continuous progression. This is demonstrated in Figure 7 which shows how proportions of identified loop types vary for an example dataset as $\eta$ is varied. It can be seen that the proportion of partial loops remains relatively stable, while changes in $\eta$ monotonically transfer cases between full and non loop classifications. Given the discussion of fit quality above, this shouldn't be surprising and, while the subjectivity of choosing a value for $\eta$ remains, the monotonicity present does offer some robustness in terms of trends identified using this method. For example, if loop sizes tend to increase with wind speed, then even with a different value of $\eta$ this result would still hold, it would just be the proportion of cases considered when observing the trend which would change. Large numbers of cases were studied in an effort to find a good value for $\eta$ which gives sensible classifications that conform to intuitive interpretations of loop type. The selected value is $\eta=0.95$. This value has performed well across test cases and, by way of example, classifies all loops in Figure 5 and the loop in Figure 4 (a) as full loops, Figure 4 (b) as a partial loop and Figure 4 (c) as a non loop.

\subsection{Applying the loop identification methodology - preliminary results}

Figures 8 shows some preliminary results from applying the developed methodology to the load dataset used in this study. In order to assess the overall prevalence of these loops during turbine operation, Figure 8 (a) shows the proportions of time spent in classification categories within each $10 \mathrm{~min}$ load time-history. From this it can be seen that proportions for each category stay relatively stable across wind speeds. Importantly, the main-bearing is experiencing either full or partial load loop events $40-50 \%$ of the time when operating. This shows these loops make up a significant proportion of turbine operational loading time histories. The benefits of a parametric characterisation of these loops for analysis purposes is demonstrated in Figure 8 (b) which shows the relationship between full loop size (captured by elliptical area) and rotor average wind speed. Given the log-scaling on the vertical axis, the observable linear trend indicates that loop sizes are increasing exponentially with wind speed. Figure 8 (c) 


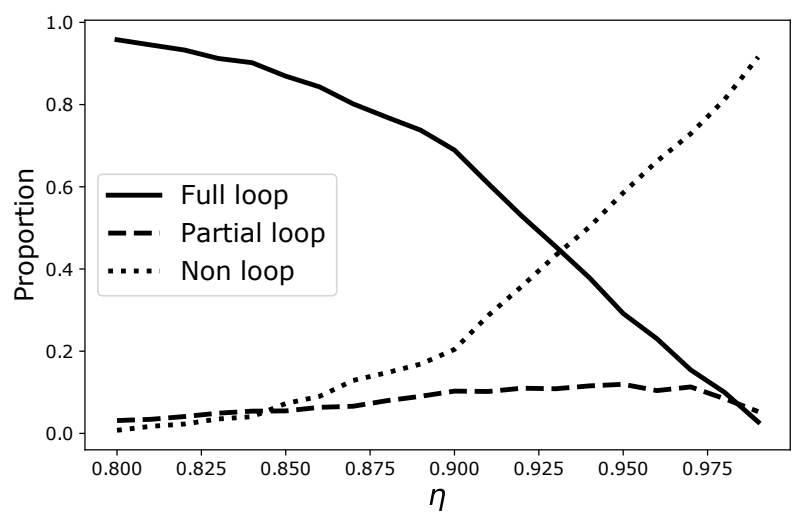

FIGURE 7 Example of proportions of identified loop types across a 10min load dataset as $\eta$ is varied.

then considers the underlying drivers for these load loops. For a low speed shaft speed of $\Omega$, wind turbines drivetrains are known to experience load fluctuations at a frequency of $3 \Omega$, owing to the symmetry of having three blades ${ }^{2}$. It therefore seems intuitive that repeated load patterns at a point on the shaft might also manifest at this same frequency. Having identified full loops, one can determine the angular speed at which that loop was traversed about its centre, denoted 'loop speed', using initial and final timestamp values. These are plotted as a histogram along with $3 \Omega$ values for the same times. Loop speeds can be seen to be heavily concentrated about $3 \Omega$, indicating that at least a proportion of full loops in loading are the result of deterministic structures in the wind field. This being the case, loops will be present for all wind turbines irrespective of control strategy or other operational factors. Finally, while these loops are observed in radial loads, an analysis was performed to determine if they have any observable relationship with thrust. Correlations between radial load magnitudes and loop size with thrust were each calculated across load datasets with results shown in Figure 8 (d). Results indicate these factors are independent of thrust, a finding which is consistent with Lavely (2017) $)^{8}$ who found out-of-plane rotor bending moments (an important contributing term in main-bearing loads) to be independent of thrust.

In the context of wider main-bearing research, as discussed in Section 1 the presented characterisation of main-bearing loading can be seen to offer a number of useful applications. The first is that the proportions and sizes of different loop types associated with wind conditions for a given site form a metric against which failure rates can be compared in order to look for correlations. At this stage it is not known what the result of such a study would be, however, the fact that the parametric approach directly captures load conditions at the main-bearing makes it an attractive candidate. In addition, the finding that a significant proportion of operational time is spent in looped load conditions also has utility with regards to model based analysis. High complexity models in which realistic effects of loading can be studied in detail are often computationally expensive and, hence, there is a limit to the number of cases and duration of loading which can reasonably be studied. Findings presented here indicate that such analysis can to some extent be restricted to studying effects of individual load loops of relevant sizes for the turbine/wind conditions at hand, thus systematising such studies and reducing the necessary workload.

\section{I AN INTERNAL LOAD MODEL FOR DOUBLE-ROW SPHERICAL ROLLER BEARINGS}

This section derives a simple and accessible internal load model for double-row spherical rollers bearings. These derivations combine aspects of both Harris and Kotzalas (2006) ${ }^{9}$ and Ghalamchi et. al. (2013) ${ }^{5}$, taking the integral load formulations of the former and reducing model dimensionality by one with respect to the latter by constraining analysis to the quasi-static case. In these previous works, roller stiffness approximations are used which have been shown to give reasonable results under certain conditions. These conditions have been found not to hold in the wind turbine case, as will be discussed below, and so a more recent and improved stiffness approximation is applied here instead.

\section{1 | Distributed loads}

This section will derive a model which solves for the continuous load distribution about the bearing circumference for a double-row spherical roller bearing under combined axial and radial loads. A single bearing row is considered first, before expanding to the double row case. 


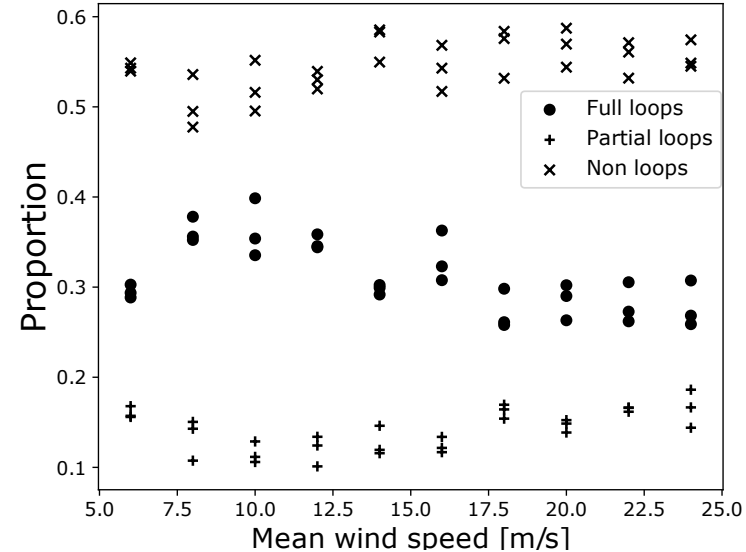

(a)

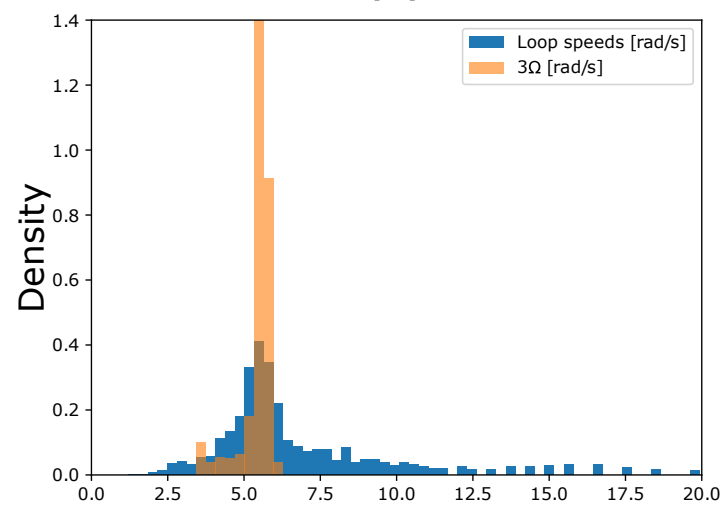

(c)

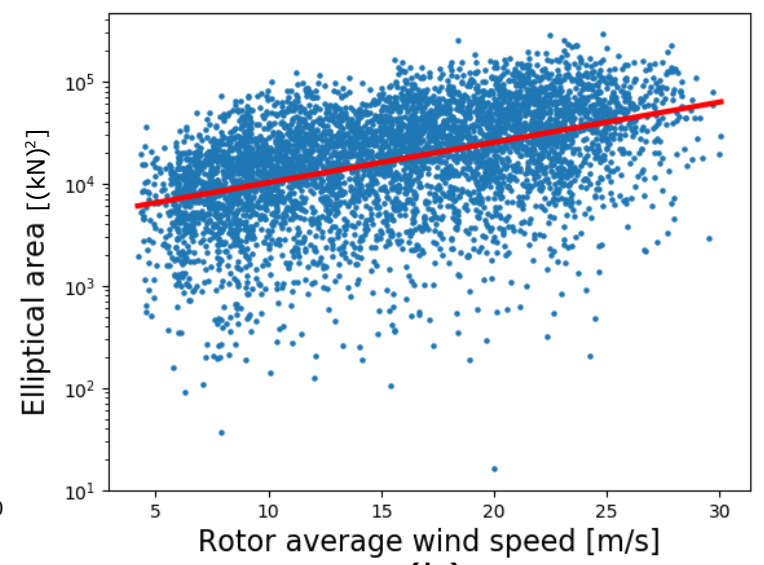

(b)

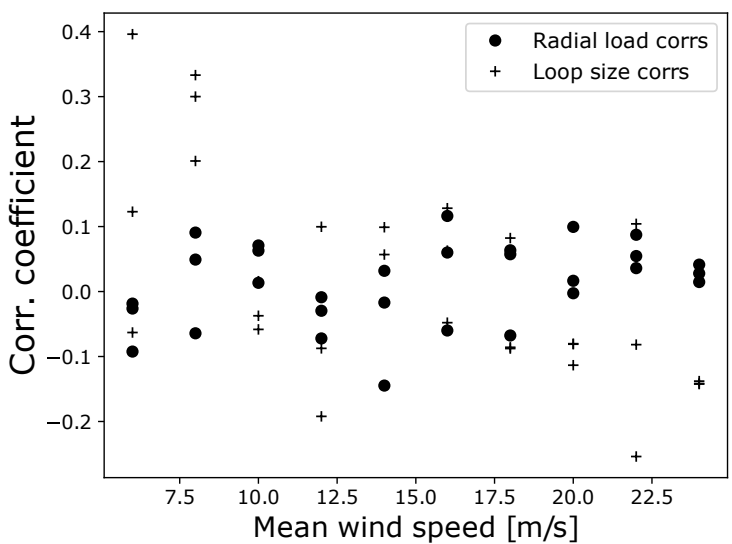

(d)

FIGURE 8 A selection of initial results from applying the loop identification methodology.

Figure 9 shows displacement geometries present in a spherical roller bearing row under combined radial and axial loads. Note that changes in contact angle, $\alpha$, are assumed to be negligible. In this case the approach of the bearing inner and outer ring: $\delta_{\psi}$, at an angle $\psi$ about the circumference can be expressed as ${ }^{9}$,

where maximum deflection (at $\psi=0$ ) is,

$$
\delta_{\psi}=\delta_{\max }\left[1-\frac{1}{2 \varepsilon}(1-\cos \psi)\right],
$$

$$
\delta_{\max }=\delta_{a} \sin \alpha+\delta_{r} \cos \alpha,
$$

and,

$$
\varepsilon=\frac{1}{2}\left(1+\frac{\delta_{a} \tan \alpha}{\delta_{r}}\right),
$$

gives the proportion of bearing circumference under load (as projected onto the diameter line incident with $Q_{\max }$ ). A roller sitting between raceways and undergoing deformation acts as a compression only nonlinear spring, with the reaction force for spherical rollers (from deformation $\delta$ ) taking the form ${ }^{9}$,

$$
Q=K_{\text {tot }} \delta^{3 / 2} .
$$

$K_{\text {tot }}$ is the total combined stiffness of roller-raceway interfaces and depends on material properties and contact geometries. This parameter will be discussed more in Section 4.1.2 Combining equations 8 and 11 and letting $Q_{\max }=K_{\text {tot }} \delta_{\max }^{3 / 2}$, it follows that the reaction force at angle $\psi$ is,

$$
Q_{\psi}=Q_{\max }\left[1-\frac{1}{2 \varepsilon}(1-\cos \psi)\right]^{3 / 2}
$$

$\ddagger$ This value can be positive or negative depending on where about the circumference it is being considered. When positive, it is equal to the compression/deflection experienced by rollers at that point. 


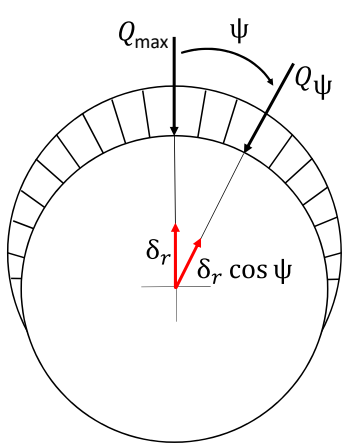

(a)

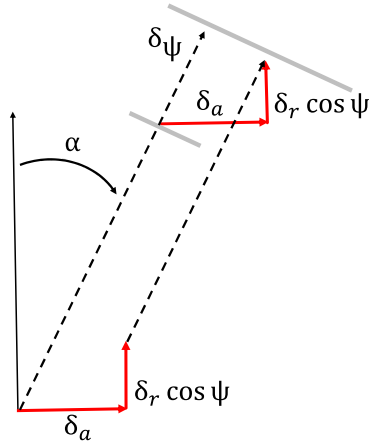

(b)

FIGURE 9 Displacement geometries in a single bearing row under combined axial and radial loads. In (b) the parallel grey lines represent the inner raceway location before and after displacement. Adapted from Harris and Kotzalas (2006) 9 .

As shown in Figure 9 it can be the case that not the whole bearing circumference is under load for a given deformation, as would be expected the limit of where rollers are under load corresponds the angle at which deformation is zero. This limiting angle, $\psi_{l}$, is determined by finding the root of Equation 8 with respect to $\psi$, resulting in,

$$
\psi_{l}=\cos ^{-1}\left(-\frac{\delta_{a} \tan \alpha}{\delta_{r}}\right)
$$

A given reaction force, $Q_{\psi}$, at some point on the bearing circumference can be resolved into radial and axial components which are respectively,

$$
Q_{\psi}^{r}=Q_{\psi} \cos \psi \cos \alpha
$$

and,

$$
Q_{\psi}^{a}=Q_{\psi} \sin \alpha .
$$

The resultant radial and axial reaction force components from all loading present around the circumference can therefore be expressed as,

$$
\begin{aligned}
Q_{r} & =Z \overline{Q_{\psi}^{r}} \\
& =\frac{Z Q_{\max } \cos \alpha}{2 \pi} \int_{-\psi_{l}}^{\psi_{l}}\left[1-\frac{1}{2 \varepsilon}(1-\cos \psi)\right]^{3 / 2} \cos \psi d \psi,
\end{aligned}
$$

and,

$$
\begin{aligned}
Q_{a} & =Z \overline{Q_{\psi}^{a}} \\
& =\frac{Z Q_{\max } \sin \alpha}{2 \pi} \int_{-\psi_{l}}^{\psi_{l}}\left[1-\frac{1}{2 \varepsilon}(1-\cos \psi)\right]^{3 / 2} d \psi,
\end{aligned}
$$

with $\mathrm{Z}$ the number of rollers present in the bearing row. Since reaction loads resulting from shaft displacements $\delta_{r}$ and $\delta_{a}$ can be evaluated, the resultant displacement under applied radial and axial loads, $G_{r}$ and $G_{a}$ respectively, can be found if the system is assumed to be at equilibrium. In this case the applied loads and bearing reaction loads will balance and hence the displacement problem can be solved by finding $\delta_{r}$ and $\delta_{a}$ values which solve,

$$
\left(G_{r}-Q_{r}\right)^{2}+\left(G_{a}-Q_{a}\right)^{2}=0 .
$$

Having determined displacements $\left(\delta_{r}^{*}, \delta_{a}^{*}\right)$ which balance the system, the load distribution about the bearing circumference takes the form,

$$
Q_{\psi}^{*}= \begin{cases}\frac{Z Q_{\max }}{2 \pi}\left[1-\frac{1}{2 \varepsilon^{*}}(1-\cos \psi)\right]^{3 / 2} & -\psi_{l}^{*}<\psi<\psi_{l}^{*} \\ 0 & \text { otherwise }\end{cases}
$$

where $\varepsilon^{*}$ and $\psi_{l}^{*}$ are those resulting from the displacements $\delta_{r}^{*}$ and $\delta_{a}^{*}$ in Equations 10 and 13 


\subsection{1 | Double-row case}

A double row bearing is now considered. Subscripts will be used to differentiate between rows, with the up-wind/rotor side row labelled 1 and the down-wind/generator side row labelled 2. Assuming the rotor lies on the left hand side of Figure 9 (b), shaft displacements $\delta_{r}$ and $\delta_{a}$ as depicted result in the following displacements for each row,

$$
\begin{gathered}
\delta_{r 1}=\delta_{r 2}=\delta_{r} \\
-\delta_{a 1}=\delta_{a 2}=\delta_{a} .
\end{gathered}
$$

The formulations of the previous section can therefore be applied within each row such that resultant radial and axial reaction forces $\left(Q_{r 1}, Q_{r 2}, Q_{a 1}, Q_{a 2}\right)$ from given shaft displacements $\left(\delta_{r}, \delta_{a}\right)$ can be evaluated. Total axial and radial reactions for the bearing as a whole then take the form,

$$
\begin{gathered}
Q_{r}=Q_{r 1}+Q_{r 2} \\
Q_{a}=Q_{a 2}-Q_{a 1}
\end{gathered}
$$

Since total reaction forces can be evaluated in the double-row case, displacements (and hence internally distributed loads) resulting from applied shaft loads can be solved for using the above root-finding approach (Equation 18 by updating the force terms in line with Equations 22 and 23 Note this analysis takes place independently of the angle at which the radial force is applied since, in the quasi-static case, deformation must be in the direction of applied load. Hence, having solved for the load distribution as above, the result is rotated such that maximum deformation and the applied load are coincident with respect to their angle about the shaft.

\subsection{2 | Determining contact and roller stiffness}

A curved surface $\mathcal{S}$ with radius of curvature $r$ along a given axis has associated with it a scalar value of curvature defined by,

$$
\rho_{\mathcal{S}}=\operatorname{sign}(\mathcal{S}) \frac{1}{r}
$$

where $\operatorname{sign}(\mathcal{S})$ is 1 for convex surfaces and -1 for concave surfaces. For two mating surfaces of revolution, denoted by I and II and with principal axes $x$ and $y$, contact geometries are captured through the quantities of curvature sum,

$$
\Sigma_{\rho}=\rho_{\mathbf{I} x}+\rho_{\mathbf{l} y}+\rho_{\mathbf{I l} x}+\rho_{\mathbf{I I} y}
$$

and curvature difference,

$$
F_{\rho}=\frac{\left(\rho_{\mathbf{l} y}-\rho_{\mathbf{I} x}\right)+\left(\rho_{\mathbf{I} y}-\rho_{\mathbf{l} x}\right)}{\Sigma_{\rho}} .
$$

Note that allocation of principal axes should be such that curvature difference is a positive quantity. In the current case the applied formulas are in fact unaffected if $F_{\rho}$ is negative due to inbuilt symmetries which will be discussed. This symmetry is specific to the particular equations used and so, as a general rule, it is recommended that the positive- $F_{\rho}$ definition is adhered to. The internal geometry of a spherical roller bearing is shown in Figure $10 D_{p}$ is bearing pitch diameter, $r_{\mathrm{Ix}}, r_{\mathrm{lx}}^{\text {in }}$ and $r_{\mathrm{Ix}}^{\text {out }}$ are contour radii and $r_{\mathrm{ly}}$ is roller radius. Remaining terms are calculated as follows,

$$
\begin{aligned}
r_{\text {lly }}^{\text {in }} & =\frac{D_{p}}{2 \cos \alpha}-r_{\text {ly }} \\
r_{\text {lly }}^{\text {out }} & =\frac{D_{p}}{2 \cos \alpha}+r_{\text {ly }} \\
\tilde{r}^{\text {in }} & =r_{\text {lly }}^{\text {in }} \cos \alpha \\
\tilde{r}^{\text {out }} & =r_{\text {lly }}^{\text {out }} \cos \alpha,
\end{aligned}
$$

assuming bearing clearance is zero. Non-zero values can be accounted for with small additions to the above $\mathrm{e}^{5}$.

The values of curvature sum and curvature difference effectively allow for analysis to be performed in the context of an equivalent ellipsoid contacting a flat plain. For the case of a point contact, an applied load results in material deformation and formation of an elliptical contact area. Letting $a$ and $b$ denote the semi-major and semi-minor axes of this contact patch respectively the ellipticity parameter, $\kappa$, is defined to be the ratio,

$$
\kappa=\frac{a}{b},
$$

which can be seen to adhere to $\kappa \geq 1$. In Hertzian theory the parameter $\kappa$ plays a key role in determining the load-deflection behaviour at a contact, with the relationship taking the form 10 ,

$$
Q=\left(\frac{\sqrt{2} \pi E}{3\left(1-\nu^{2}\right)} \kappa \sqrt{\frac{\mathcal{E}(\kappa)}{\mathcal{F}(\kappa)^{3} \Sigma_{\rho}}}\right) \delta^{3 / 2},
$$




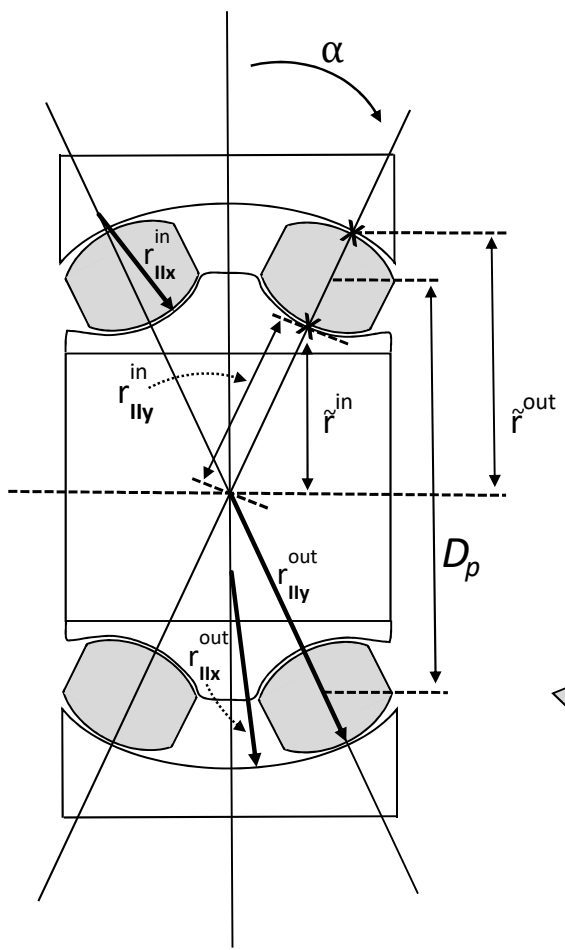

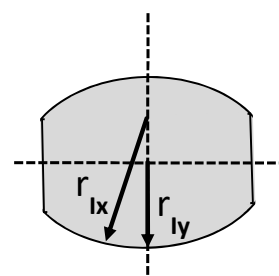

(b)

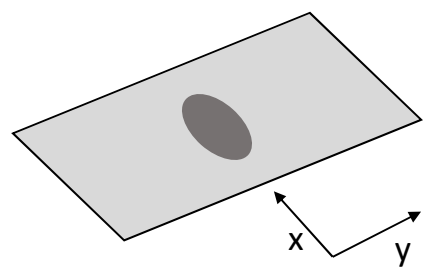

(c)

(a)

FIGURE 10 Internal geometry of a spherical roller bearing.

for contacting bodies of the same material with modulus of elasticity $E$ and Poisson's ratio $\nu$. $\mathcal{F}$ and $\mathcal{E}$ are so called elliptic integrals of the first and second kind. Both functions of $\kappa$, they are related to the geometry of the contact patch and, more specifically, to arc-length about the elliptical contact area. In the context of Equation 11 (and at this stage only for a single contact interface) the contact stiffness, $K$, is therefore,

$$
\begin{aligned}
K & =\frac{\sqrt{2} \pi E}{3\left(1-\nu^{2}\right)} \kappa \sqrt{\frac{\mathcal{E}(\kappa)}{\mathcal{F}(\kappa)^{3} \Sigma_{\rho}}} \\
& \approx 3.336 \times 10^{5} \kappa \sqrt{\frac{\mathcal{E}(\kappa)}{\mathcal{F}(\kappa)^{3} \Sigma_{\rho}}} \text { (for steel bodies). }
\end{aligned}
$$

The above approximation is arrived at using standard values of $E=2.05 \times 10^{5} \mathrm{MPa}$ and $\nu=0.3$. Note that units of $\mathrm{MPa}\left(=\mathrm{N} / \mathrm{mm}^{2}\right)$ are used in conjunction with distance units of $\mathrm{mm}$ throughout. In order to calculate contact stiffness, $K$, it therefore only remains to be able to determine values for $\kappa, \mathcal{F}(\kappa)$ and $\mathcal{E}(\kappa)$. The implicit nature of the equations which define these terms has historically meant that iterative procedures are required for their resolution. In order to avoid these time-consuming computations, a number of empirical explicit formulations have been proposed which allow for direct computation of the required terms. It has been shown ${ }^{11}$ that $\mathcal{F}$ and $\mathcal{E}$ can be computed using,

$$
\begin{aligned}
\mathcal{F}(\kappa) & =\left(\lambda_{0}+\lambda_{1} \kappa^{-2}+\lambda_{2} \kappa^{-4}\right)-\ln \left(\kappa^{-2}\right)\left(\lambda_{3}+\lambda_{4} \kappa^{-2}+\lambda_{5} \kappa^{-4}\right) \\
\mathcal{E}(\kappa) & =\left(\beta_{0}+\beta_{1} \kappa^{-2}+\beta_{2} \kappa^{-4}\right)-\ln \left(\kappa^{-2}\right)\left(\beta_{3} \kappa^{-2}+\beta_{4} \kappa^{-4}\right),
\end{aligned}
$$

valid for $\kappa \geq 1$ and with errors not exceeding 20 ppm (part per million) and 40 ppm respectively. For a given contact interface a new variable $X$ is now defined as,

$$
X=\frac{1+F_{\rho}}{1-F_{\rho}}
$$

Antoine et. al. (2006) ${ }^{11}$ show that $\kappa$ itself can be well approximated as a function of $X$ across the full range of tested surface ellipticity values $\left(1 \leq X \leq 10^{10}\right)$ using the expression $\$$

$$
\kappa(X)=X^{\operatorname{sign}\left(F_{\rho}\right) \gamma\left(\log _{10} X\right)},
$$

${ }^{\S}$ The notation used in Antoine et. al. (2006) ${ }^{11}$ was found to be somewhat difficult to follow. As such, an adjusted notation is presented here in an attempt to improve clarity. 
with,

$$
\gamma(x)=\frac{2}{3}\left(\frac{1+\mu_{1} x^{2}+\mu_{2} x^{4}+\mu_{3} x^{6}+\mu_{4} x^{8}}{1+\mu_{5} x^{2}+\mu_{6} x^{4}+\mu_{7} x^{6}+\mu_{8} x^{8}}\right) .
$$

Coefficient values for the above expressions are given in Antoine et. al. (2006) 11 . Note, the use of a logarithm combined with $\gamma$ being an even function removes sensitivity to the sign of $F_{\rho}$; in the original work ${ }^{11}$ this only extended as far as the function $\gamma$, whereas the additional sign $\left(F_{\rho}\right)$ term included here extends this desirable property to the whole $\kappa$ expression. As noted above, this formulation has been shown to provide high accuracy over a wide range of ellipticity values. The approximations used in other work ${ }^{510}$ on the other hand have been shown ${ }^{12}$ to perform well for $1 \leq \kappa \leq 10$, but, in the wind turbine case normal values for $\kappa$ are found to be around 40 . Therefore, the improved formulations as presented here are necessary.

The above allows contact stiffness for a given interface between approaching surfaces to be evaluated. With respect to roller bearings there will in fact always be two interfaces, one between roller and inner raceway and one for the outer raceway, whose load-deflection behaviours need to be accounted for. With inner and outer contact stiffness values $K_{i}$ and $K_{o}$ respectively, given by Equation 33 and associated with the deflections $\delta_{o}$ and $\delta_{i}$, the roller becomes a series of two non-linear springs. Hence, one would like to find an equivalent spring stiffness, $K_{\text {tot }}$, which can be used to represent the whole system. Due to the fact that forces within a series of springs must be equal throughout, this is equivalent to solving for $K_{\text {tot }}$ such that,

$$
K_{i} \delta_{i}^{3 / 2}=K_{o} \delta_{o}^{3 / 2}=K_{\text {tot }}\left(\delta_{i}+\delta_{o}\right)^{3 / 2}
$$

resulting in,

$$
K_{\text {tot }}=\left(\frac{1}{\left(\frac{1}{K_{i}}\right)^{2 / 3}+\left(\frac{1}{K_{o}}\right)^{2 / 3}}\right)^{3 / 2} .
$$

This value, $K_{\text {tot }}$, is the total combined stiffness for roller-raceway interfaces used in Equation 11 in Section 4.1. In practise it is the total roller deformation, $\delta$, which is solved for. While this is equal to the sum of inner and outer contact deformations, $\delta=\delta_{i}+\delta_{o}$, for the current work (and in general) these component values themselves are not required and one works directly with $\delta$ and $K_{\text {tot. }}$.

\section{2 | Individual roller loads}

Consider a load distribution for bearing row $i, Q_{\widetilde{\psi} i}$, for an angle $\widetilde{\psi} i$ in the shaft reference frame (i.e. post-rotation to be coincident with load direction). The current formulation will assume rollers are evenly spaced around the bearing circumference. In this case, for a bearing containing $Z$ rollers per row, angular spacing is,

$$
\widetilde{\psi}_{\dagger}=\frac{2 \pi}{Z} \text {. }
$$

Each roller is assumed to carry the portion of distributed load lying closer to its own roller centre than to any other. The carried load for a roller in row $i$ at angular position $\widetilde{\psi}_{\text {roll }}$ is therefore obtained by integrating the distribution through the relevant arc,

$$
Q_{\text {roll }, i}\left(\widetilde{\psi}_{\text {roll }}\right)=\int_{\widetilde{\psi}_{\text {roll }}-\widetilde{\psi}_{\dagger} / 2}^{\widetilde{\psi}_{\text {roll }}+\widetilde{\psi}_{\dagger} / 2} Q_{\widetilde{\psi} i} d \tilde{\psi} .
$$

Similar integrals taken over remaining arc sections result in roller load values across the full complement of rollers. Now that individual roller loads can be extracted from the distributions, if the positions of rollers during operation can be tracked or modelled (independently of the forcebalance/displacement-solving procedure) then time-variations in roller loads can be studied without needing to re-run or augment the models of Section 4.1. This can be approached in a number of ways, with the most straightforward considered in the following section. Note that integrals of the form of Equation 43 can also be used to extract roller loads from more general or changing roller spacings if required.

\subsection{1 | Roller orbital trajectories}

Having determined a time history (in the quasi-static sense) for bearing load distributions in Section 4.1 and also being able to extract individual roller loads based on roller angular positions, Section 4.2 in order to extract time series of load for individual rollers all that is required is the trajectory of rollers about the bearing centre. As has been outlined, the formulations of loading into distributions was specifically to allow for modularity between load analyses and roller trajectories since:

1. Load distributions themselves may be enough information for given applications and hence a method which can obtain this without needing to track roller positions avoids adding unnecessary complexity and computation

2. True roller trajectories are complex and nonlinear with multiple interactions and, hence, various models can be applied. The modularity of the approach developed here means load distributions can be obtained first, with such effects accounted for later, as required. This can be 
in the form of a simple 'no-slip' assumption, as will be applied here, or by extension to include a more sophisticated dynamic model 644 to interact with force distributions.

In the current work, the simplest of these is used to allow characteristics of loading seen by individual rollers to be examined.

Bearing internal geometry was shown in Figure 10 In this case, orbital roller speeds can be determined as follows: Let the rotational speed of the inner race/shaft be denoted $\Omega_{i}$, with the outer race assumed to be stationary. As the inner raceway rotates the roller is assumed to move without slip at the roller-raceway contact points. Therefore, instantaneous tangential velocities for raceways and roller surfaces must be equal at contact points, with common velocities,

$$
v_{i}=\Omega_{i} \tilde{r}^{\text {in }}, \quad v_{o}=0
$$

The instantaneous tangential velocity at the centre (denoted by subscript c) of a roller must then equal the mean of these two values,

$$
v_{c}=\frac{v_{i}+v_{o}}{2}=\frac{\Omega_{i} \tilde{r}^{\text {in }}}{2} .
$$

It follows that the orbital speed of the roller, $\Omega_{c}$, about the bearing centre is,

$$
\Omega_{c}=\frac{v_{c}}{\tilde{r}_{c}}=\frac{\Omega_{i}}{2}\left(\frac{\tilde{r}^{\text {in }}}{\tilde{r}^{\text {in }}+\frac{\tilde{r}^{\text {out }}-\tilde{r}^{\text {in }}}{2}}\right) .
$$

The term $\left(\tilde{r}^{\text {out }}-\tilde{r}^{\text {in }}\right) / 2$ above will generally be small with respect to bearing dimensions and so the latter term in Equation 46 can be seen to approximately equal 1 . Hence, $\Omega_{c} \approx \Omega_{i} / 2$, which is a useful rule of thumb 1 when considering the impacts of identified MB load structures.

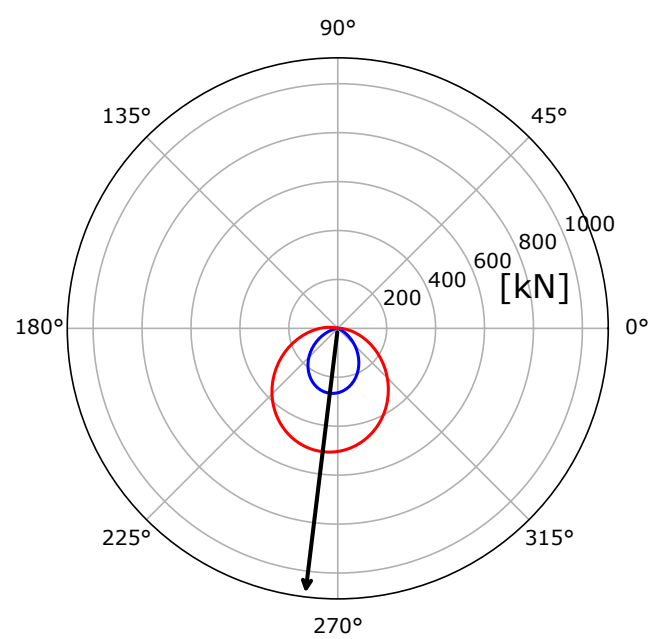

FIGURE 11 Distributed load example from an applied load (black) on the upwind (blue) and downwind (red) bearing rows.

Having developed an expression for roller orbital speed one can then calculate trajectory, $T(t)$, at any time $t$ after start time $t_{0}$ via integration,

$$
T(t)=\int_{t_{0}}^{t} \Omega_{c}\left(t^{\prime}\right) d t^{\prime} .
$$

In the current case, the assumption of equally distributed rollers means the trajectory for only a single roller need be tracked, with positions for the others obtainable via addition of relevant separation angles.

\section{5 | IMPACTS OF IDENTIFIED LOAD STRUCTURES ON MAIN-BEARING INTERNAL LOADS}

This sections considers time-varying loads experienced by rollers in a double row spherical roller bearing via application of the theory presented in Section 4 In particular, it is important to establish whether the identified load structures of Section 2 result in observable impacts to roller loads

T This approximation is used to aid intuition and discussion only, with the full expression in Equation 46 applied in practise. 


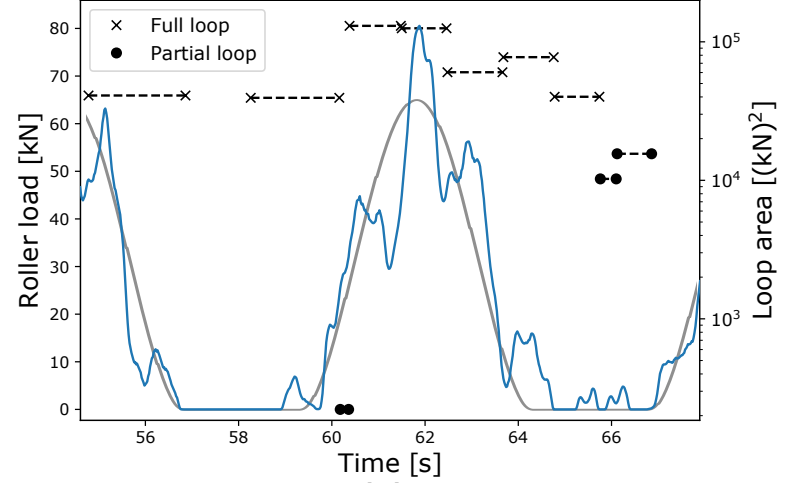

(a)

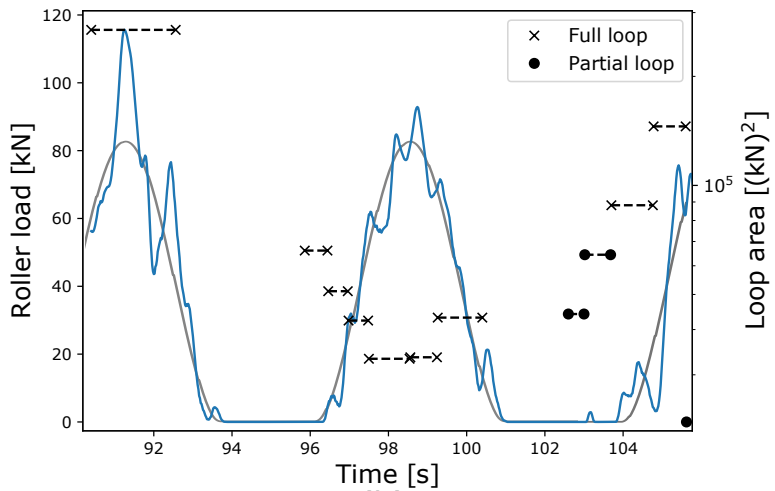

(b)

FIGURE 12 Roller loading examples (blue) under turbulent wind loading from simulations in mean wind speeds of 18 and $24 \mathrm{~m} / \mathrm{s}$ are shown in (a) and (b) respectively. Roller loading from a constant applied main-bearing load are shown in grey for comparison.

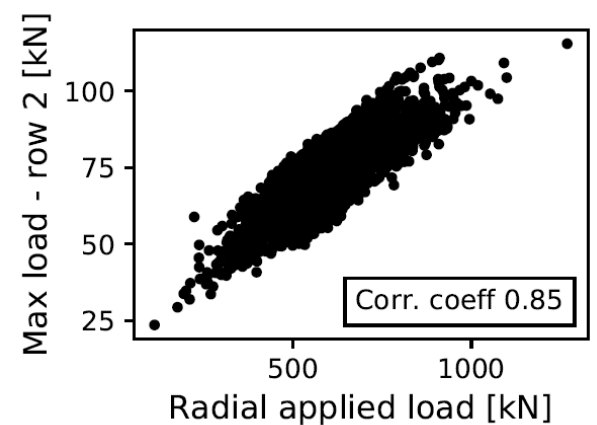

(a)

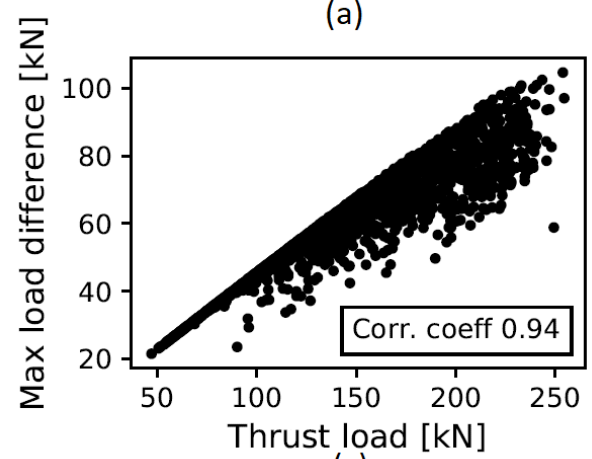

(c)

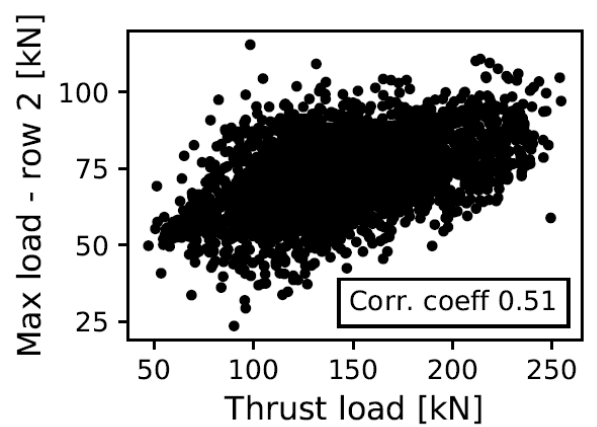

(b)

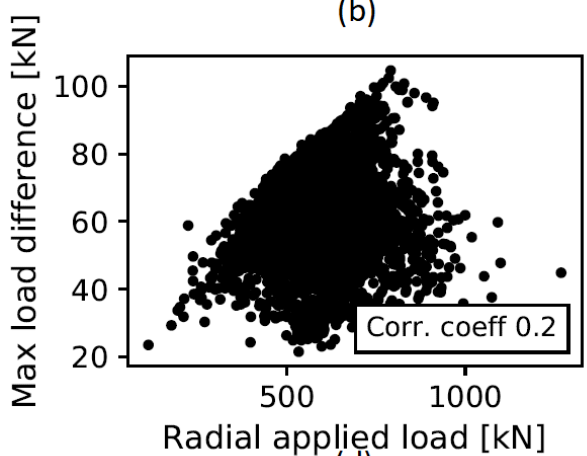

(d)

FIGURE 13 Correlation analysis between applied main-bearing loads and resultant roller loads.

such that further study of these load phenomena is warranted. Analysis was performed using a distributed load model representation of an SKF 240/630CA/W33 bearing which have been used in wind turbines of this size.

Figure 11 shows a typical distributed load output. As can be seen, the downwind row takes most of the load (due to thrust), with the upwind row only lightly loaded. Results will therefore concentrate on rollers in the downwind row. Figure 12 shows time-series of loading for an individual downwind roller during operation in turbulent wind fields and, for comparison, under constant load. In addition, concurrent identified full and partial loops from the applied loads are plotted with loop area forming a second vertical axis. In these plots it can clearly be seen that identified looped structures in loading correspond closely to significant fluctuations in roller loads. Loop areas also appear to dictate the size of roller load fluctuations, with the largest loops appearing alongside the largest deviations from the constant load profiles. In the analysis of Section 4.2 .1 it was shown that roller orbital speeds are approximately $\Omega_{i} / 2$ which, combined with the results of Section 3.3 that indicate looping speeds are concentrated about $3 \Omega_{i}$, implies a factor of six between roller orbital speeds and looping speeds. As such, one would expect to see multiple loop events per revolution of a roller about the shaft, as is the case in these examples. 
With regard to possible impacts on bearing health from time varying loads in Figure 12 there will be a clear contribution to surface fatigue damage imparted by observable fluctuations, as well as possible excursions to damaging peak loads. In addition, load loops can be seen to result in load fluctuations within the unloaded zone. The reason this may be important is due to the potential for abrasive and adhesive wear from bearing skidding 413 (a combination of rolling and sliding) known to occur as a roller becomes loaded \# under steady load these events occur once per rotation 6 , however, results presented here indicate rollers will experience multiple loading and unloading events per rotation. This suggests damage associated with such events may be a driver in understanding premature main-bearing failures. Recall that the current model applies a no-slip assumption to evaluate roller trajectories and so slip events would result in deviations from the exact load profiles seen here. However, incidents of slip will arguably only exacerbate observed effects since roller orbital speeds will on average be slower and hence, if anything, rollers would experience greater numbers of loading-unloading events during each orbit. The presented results indicate that identified load loops do significantly impact roller loading within the bearing, even in the presence of thrust. The fact that loop events can be seen to correspond closely to fluctuations in roller loads also motivates the possibility that the loop identification methodology may be sensibly extended to act as a damage equivalent load type descriptor, with the benefit that it doesn't assume any particular damage type and so could be tied to both surface fatigue based damage as well as the prevalence of other effects such as skidding.

The results presented above were found to hold generally across tested cases, with thrust seen to mainly drive load sharing between rows 1 and 2, and radial forces being the main driver for roller load fluctuations over short-timescales. To put these claims on a firmer footing, a correlation analysis was performed between applied radial and thrust loads and the maximum roller load in row 2 (downwind) as well as the difference in maximum roller load between rows. Results for this analysis are shown in Figure 13 Radial loading can be seen to correlate strongly with maximum roller load, whereas only a weak relationship exists in the case of thrust loading. Similarly a strong correlation exists between maximum load differences and thrust, whereas no discernible relationship is found in the radial load case. These findings are consistent with the observations that thrust drives load sharing between rows and radial loads are the main driver for load changes within each row.

Taken together, the findings of this section indicate:

- Load loops identified in applied radial loads have significant impact on bearing roller loads

- Radial loads are the stronger driver for individual roller load fluctuations, hence loop impacts remain important in the presence of thrust

- Observable likely implications from the presence of load loops include increased surface fatigue and peak roller loads as well as possible abrasive/adhesive wear from bearing skid events.

\section{6 | CONCLUSIONS}

This paper has described the presence of looped structures in wind turbine main-bearing radial loads resulting from repeating patterns of fluctuation in radial load magnitudes and directions. An approach was developed which allows such structures to be identified, classified and studied. Results from the application of these methods were presented, with the main findings being: the areas (i.e. sizes) of identified load loops tend to increase with wind speed, loop frequencies are centred about the rotor ' $3 \Omega$ ' frequency and hence linked to deterministic wind field structures and, finally, roughly $50 \%$ of operational time is spent in some form of looped load structure. A distributed load model for double-row spherical roller bearings was also developed in order to ascertain likely impacts of such loading on bearing roller loads and to assess whether these loads remain important in the presence of thrust. Results indicate that identified load loops have significant impacts on roller loading, with possible effects which should be considered in future work including surface fatigue damage contributions, harmful peak loading and skidding events caused by multiple instances of roller loading and unloading per revolution. Findings motivate further study of these load phenomena using the methods developed in this paper.

\section{ACKNOWLEDGEMENTS}

The author would like to thank ONYX InSight for their support and advice throughout this work. Thanks are also extended to Alasdair McDonald and David Infield for their valuable comments and suggestions. Finally, the author would like to acknowledge the financial support of the EPSRC under grant number EP/R513349/1.

Code and data relating to this work will be made available. For access please contact the author directly. In addition, a toolbox of presented methods is in preparation and will be made available at https://pureportal.strath.ac.uk/en/persons/edward-john-hart once complete.

\# The larger fluctuations through the loaded zone may also result in important dynamic effects but, once loads are high enough, rollers tend not to skid since frictional forces overcome roller inertia. 


\section{References}

1. Hart E, Turnbull A, Feuchtwang J, McMillan D, Golysheva E, Elliott R. Wind turbine main-bearing loading and wind field characteristics. Wind Energy 2019; 22: 1534-1547. doi: https://doi.org/10.1002/we.2386

2. Hart E, Clarke B, Nicholas G, et al. A review of wind turbine main bearings: design, operation, modelling, damage mechanisms and fault detection. Wind Energy Science 2020; 5(1): 105-124. doi: 10.5194/wes-5-105-2020

3. Zheng J, Ji J, Yin S, Tong VC. Internal loads and contact pressure distributions on the main shaft bearing in a modern gearless wind turbine. Tribology International 2020; 141: 105960. doi: https://doi.org/10.1016/j.triboint.2019.105960

4. Han Q, Li X, Chu F. Skidding behavior of cylindrical roller bearings under time-variable load conditions. International Journal of Mechanical Sciences 2018; 135: 203 - 214. doi: https://doi.org/10.1016/j.ijmecsci.2017.11.013

5. Ghalamchi B, Sopanen J, Mikkola A. Simple and versatile dynamic model of spherical roller bearing. Hindawi Publishing Corporation International Journal of Rotating Machinery 2013. doi: 10.1155/2013/567542

6. Jain S, Hunt H. A dynamic model to predict the occurrence of skidding in wind-turbine bearings. Journal of Physics: Conference Series 2011; 305: 012027. doi: 10.1088/1742-6596/305/1/012027

7. Fitzgibbon A, Pilu M, Fisher R. Direct Least-squares fitting of ellipses. Pattern Analysis and Machine Intelligence, IEEE Transactions on 1996; 21: 253 - 257 vol.1. doi: 10.1109/ICPR.1996.546029

8. Lavely A. Effects of daytime atmospheric boundary layer turbulence on the generation of nonsteady wind turbine loadings and predictive accuracy of lower order models. PhD thesis. Pennsylvania State University, 2017.

9. Harris T, Kotzalas M. Essential Concepts of Bearing Technology. Rolling Bearing Analysis, Fifth Edition, Chapter 7, CRC Press . 2006.

10. Harris T, Kotzalas M. Essential Concepts of Bearing Technology. Rolling Bearing Analysis, Fifth Edition, Chapter 6, CRC Press . 2006.

11. Antoine JF, Visa C, Sauvey C, Abba G. Approximate analytical model for Hertzian elliptical contact problems. Journal of Tribology-Transactions of the ASME 2006; 128. doi: 10.1115/1.2197850

12. Brewe DE, Hamrock BJ. Simplified Solution for Elliptical-Contact Deformation Between Two Elastic Solids. Journal of Lubrication Technology 1977; 99(4): 485-487. doi: 10.1115/1.3453245

13. Bujoreanu C, Cretu S, Nelias D. Scuffing Behaviour in angular contact ball bearings. Annals of Dunarea de Jos University of Galati 2003 ; Il: $33-39$.

How to cite this article: Hart E, (2020), Developing a systematic approach to the analysis of time-varying main-bearing loads for wind turbines, Wind Energy, $x x x$.

\section{APPENDIX}

\section{A - ELLIPSE FITTING METHOD OVERVIEW}

The ellipse fitting method used in the current work is that of Fitzgibbon et. al. (1996)7! A brief summary of this method is as follows: For a general conic defined implicitly by the equation,

$$
\mathcal{C}(\boldsymbol{\sigma}, \mathbf{y})=\boldsymbol{\sigma} \cdot \mathbf{y}=\sigma_{1} y^{2}+\sigma_{2} y z+\sigma_{3} z^{2}+\sigma_{4} y+\sigma_{5} z+\sigma_{6}=0,
$$


with $\boldsymbol{\sigma}=\left[\sigma_{1}, \ldots, \sigma_{6}\right]^{T}$ and $\mathbf{y}=\left[y^{2}, y z, z^{2}, y, z, 1\right]^{T}$. An ellipse is represented when the discriminant, $\sigma_{2}^{2}-4 \sigma_{1} \sigma_{3}$, is negative. For measured data $\left\{\mathbf{y}_{i}\right\}$ let $D=\left[\mathbf{y}_{1}, \ldots, \mathbf{y}_{N}\right]^{T}$ and

$$
C=\left[\begin{array}{cccccc}
0 & 0 & 2 & 0 & 0 & 0 \\
0 & -1 & 0 & 0 & 0 & 0 \\
2 & 0 & 0 & 0 & 0 & 0 \\
0 & 0 & 0 & 0 & 0 & 0 \\
0 & 0 & 0 & 0 & 0 & 0 \\
0 & 0 & 0 & 0 & 0 & 0
\end{array}\right],
$$

(where $C$ encodes the discriminant condition) the coefficients corresponding to the least squares elliptical fit are given by the only eigenvector of $M=\left(D^{T} D\right)^{-1} C$ having a positive eigenvalue.

\section{B - COEFFICIENT OF DETERMINATION}

The coefficient of determination, commonly denoted $R^{2}$, describes the proportion of variance present in a set of measured values which is captured by a model fitted to the data. Explicitly, let $\mathbf{P}$ and $\mathbf{Q}$ each contain $n$ points in two-dimensions,

$$
\begin{gathered}
\mathbf{P}=\left[\begin{array}{cc}
p_{1}^{y} & p_{1}^{z} \\
p_{2}^{y} & p_{2}^{z} \\
\vdots & \vdots \\
p_{n}^{y} & p_{n}^{z}
\end{array}\right] \\
\mathbf{Q}=\left[\begin{array}{cc}
q_{1}^{y} & q_{1}^{z} \\
q_{2}^{y} & q_{2}^{z} \\
\vdots & \vdots \\
q_{n}^{y} & q_{n}^{z}
\end{array}\right] .
\end{gathered}
$$

The total sum of squares for data $\mathbf{P}$ is defined as,

$$
S_{\text {total }}(\mathbf{P})=\sum_{i=1}^{n}\left(p_{i}^{y}-\overline{p^{y}}\right)^{2}+\left(p_{i}^{z}-\overline{p^{z}}\right)^{2},
$$

and the residual sum of squares for model $\mathbf{Q}$ as a fit to data $\mathbf{P}$ as,

$$
S_{\text {resid }}(\mathbf{Q}, \mathbf{P})=\sum_{i=1}^{n}\left(p_{i}^{y}-q_{i}^{y}\right)^{2}+\left(p_{i}^{z}-q_{i}^{z}\right)^{2} .
$$

The $R^{2}$ value for model $\mathbf{Q}$ as a fit to data $\mathbf{P}$ is then given by,

$$
R^{2}(\mathbf{Q}, \mathbf{P})=1-\frac{S_{\text {resid }}(\mathbf{Q}, \mathbf{P})}{S_{\text {total }}(\mathbf{P})} .
$$

